\title{
Momentum-space engineering of gaseous Bose-Einstein condensates
}

\author{
Mark Edwards, ${ }^{1,2}$ Brandon Benton, ${ }^{1}$ Jeffrey Heward,${ }^{1}$ and Charles W. Clark ${ }^{3}$ \\ ${ }^{1}$ Department of Physics, Georgia Southern University, Statesboro, GA 30460-8031 USA \\ ${ }^{2}$ National Institute of Standards and Technology, Gaithersburg, MD 20899, USA \\ ${ }^{3}$ Joint Quantum Insitute, National Institute of Standards and Technology \\ and the University of Maryland, Gaithersburg, MD 20899, USA
}

(Dated: November 13, 2018)

\begin{abstract}
We show how the momentum distribution of gaseous Bose-Einstein condensates can be shaped by applying a sequence of standing-wave laser pulses. We present a theory, whose validity for was demonstrated in an earlier experiment [L. Deng, et al., Phys. Rev. Lett. 83, 5407 (1999)], of the effect of a two-pulse sequence on the condensate wavefunction in momentum space. We generalize the previous result to the case of $N$ pulses of arbitrary intensity separated by arbitrary intervals and show how these parameters can be engineered to produce a desired final momentum distribution. We find that several momentum distributions, important in atom-interferometry applications, can be engineered with high fidelity with two or three pulses.
\end{abstract}

PACS numbers: 03.75.Gg,67.85.Hj,03.67.Dg

\section{INTRODUCTION}

The ability to create gaseous Bose-Einstein condensates in the laboratory [1 [5] has led to new vistas in the field of atom interferometry. This is particularly true when laser light is used to manipulate atoms to produce matter-wave interference patterns. Atom interferometers using light gratings acting on matter waves have been used in a variety of fundamental studies such as how large a composite object can display interference effects [6], decoherence studies [7, 8], origins of phase shifts under various circumstances, properties of Bose-Einstein condensates 9 11], and testing the charge neutrality of atoms 12]. Atom interferometers are also at the heart of a host of practical devices used for making precision measurements. These include gravimeters, gyroscopes, and gradiometers all of which have important applications in precision navigation [13 15]. Such interferometers also have applications in atomic physics such as atomic polarizability measurements and Casimir-Polder potentials for atoms near surfaces [16]. More in-depth information about the uses of atom interferometry can be found in Ref. [17].

One of the crucial elements of an atom interferometer is initial state selection of the atoms and these states are generally states of localized momentum [17. Momentum-state selection techniques are quite varied and can range from using a pair of collimating slits that select thermal atoms with limited transverse momentum to Bose-Einstein condensation of atom clouds via techniques that are a combination of laser cooling and trapping and evaporative cooling. In this paper we show how applying a sequence of short-time, standing-wave laser pulses to a Bose-Einstein condensate (BEC) can be used as a tool for the state selection step of a atom interferometric experiment. We will also see that preparation of several important classes of momentum states can be achieved through the application of just two or three pulses.
Previously a sequence of two short-time, standingwave pulses (sometimes called Kapitza-Dirac pulses) has been used as the beam splitter in the experimental realization of a Michelson atom interferometer for a BEC formed on an atom chip [18]. Optimization of the twopulse sequence was determined by studying a two-state truncation of the Raman-Nath equations [19]. Other related beam-splitter techniques which produce specific momentum orders 20 22] have been studied. However, these techniques represent a different strategy [19] in the standing-wave control of atomic motion.

This paper is organized as follows. In Section II, we derive a theory for how the condensate wave function is changed following the application of two pulses of different intensity and separated by a time interval on the order of the Talbot time. The Talbot time is $T_{T}=h / E_{\text {recoil }}$ where $E_{\text {recoil }}$ is the recoil energy of the atom for the laser light used to make the standing-wave pulses. This theory is composed of two parts, (1) the effect of a pulse on the condensate wave function and (2) evolution of the wave function between pulses. These two elements are used to follow the steps of a two-pulse sequence to derive an expression for the amplitude for atoms to jump from the zero-momentum initial state to an arbitrary momentum order. In Section [II] we derive two general symmetries of the two-pulse amplitude and some values for special time interval values. This section also presents a physical interpretation of the amplitude. This interpretation is especially useful for generalizing the two-pulse result. Section [V] contains a derivation of the general $N$-pulse amplitude. Section $\nabla$ presents the least-squares method for designing a general sequence of $N$ pulses which gives a specified momentum probability distribution. Examples of two-pulse and three-pulse sequences are given for some important momentum distributions. Conclusions are presented in Section VI 


\section{TWO-PULSE THEORY}

In this section we derive how the condensate wave function is changed when two short-time, standing-wave laser pulses are applied. We assume that each pulse is square-shaped in time and that its duration, $\delta t$, is short enough such that there is no appreciable spontaneous emission during the pulse and short enough so that are no effects of atom-atom interactions during the pulse. This will happen if $\delta t<\hbar / \Gamma$ where $\Gamma$ is the natural line width of the excited state. We also assume that the entire pulse sequence is short compared to $\hbar / \mu$, where $\mu$ is the chemical potential, i.e., short enough so that there are no effects due to the interaction during the pulse sequence. It is important to note that, under these assumptions, many-body effects can be neglected.

We assume that the first pulse is applied at time $t_{1}$ and has duration $\delta t_{1}$ and that the second pulse is applied at time $t_{2}$ and has duration $\delta t_{2}$. We also assume that the time interval, $t_{2}-t_{1}$, is small enough so that the maximum distance that condensate atoms in nonzero momentum states move after the first pulse is small compared to their de Broglie wavelengths. This is the Raman-Nath regime.

Our approach will be to use these conditions to approximate the effect that a single pulse has on the condensate wave function and separately to approximate how the wave function evolves between pulses. With these effects in hand, we can then follow the steps of the pulse sequence to determine the overall effect of the full pulse sequence on the wave function.

\section{A. Effect of the first pulse}

Consider atoms in a Bose-Einstein condensate interacting with a pair of linearly polarized, counterpropagating laser pulses. Each atom is modeled as a two-level system having a ground state $|g\rangle$ and an excited state $|e\rangle$. We denote the position of the atom's center-of-mass (CM) relative to an arbitrary coordinate system by $\mathbf{r}_{a}$ and the position of the atomic electron relative to the CM by $\mathbf{r}_{e}$ so that the position vector of the electron in the arbitrary system is $\mathbf{r}_{a}+\mathbf{r}_{e}$.

The Hamiltonian for a single atom interacting with the light and including the CM motion is given by

$$
\begin{aligned}
H= & H_{0}^{(g)}\left(\mathbf{r}_{a}\right)|g\rangle\left\langle g\left|+H_{0}^{(e)}\left(\mathbf{r}_{a}\right)\right| e\right\rangle\langle e| \\
& +E_{g}|g\rangle\left\langle g\left|+E_{e}\right| e\right\rangle\langle e|+V_{\text {laser }}\left(\mathbf{r}_{a}, t\right) .
\end{aligned}
$$

Where

$$
H_{0}^{(k)}\left(\mathbf{r}_{a}\right)=\frac{\mathbf{p}_{a}^{2}}{2 m}+V_{\text {trap }}^{(k)}\left(\mathbf{r}_{a}\right), \quad k=g, e
$$

are the energies associated with the CM motion of the atom in the ground and excited states. The difference in trap potentials derives from the different magnetic moments for the two internal states. Both potentials are assumed to be harmonic here.
The term $V_{\text {laser }}$ is the usual dipole interaction and is written as

$$
\begin{aligned}
V_{\text {laser }}\left(\mathbf{r}_{a}, t\right)= & 2 \hbar \Omega_{0} \cos \left(\mathbf{k}_{L} \cdot \mathbf{r}_{a}\right) f(t) \cos \left(\omega_{L} t\right) \\
& \times(|e\rangle\langle g|+| g\rangle\langle e|)
\end{aligned}
$$

where the standing-wave laser field is assumed to have the form

$$
\mathbf{E}\left(\mathbf{r}_{a}, t\right)=2 \mathcal{E}_{0} f(t) \hat{\mathbf{e}}_{L} \cos \left(\mathbf{k}_{L} \cdot \mathbf{r}_{a}\right) \cos \left(\omega_{L} t\right) .
$$

The laser-field amplitude, frequency, and wavevector are denoted by $\mathcal{E}_{0}, \omega_{L}$, and $\mathbf{k}_{L}$, respectively, while $\hat{\mathbf{e}}_{L}$ is the laser polarization vector. The laser-field amplitude envelope, $f(t)$, is assumed to be a rectangular pulse centered at an arbitrary time $t_{1}$ with width $\delta t_{1}$. The factor $\Omega_{0}$ in Eq. (3) is the single-photon Rabi frequency given by

$$
\hbar \Omega_{0}=e \mathcal{E}_{0}\left\langle e\left|\mathbf{r}_{e} \cdot \hat{\mathbf{e}}_{L}\right| g\right\rangle
$$

where $e$ is the electron charge.

The condensate orbital is represented by a spinor wave function of the form

$$
\begin{aligned}
\Psi\left(\mathbf{r}_{a}, t\right) & =\left(\begin{array}{c}
\psi_{g}\left(\mathbf{r}_{a}, t\right) \\
\psi_{e}\left(\mathbf{r}_{a}, t\right)
\end{array}\right) \\
& =\psi_{g}\left(\mathbf{r}_{a}, t\right)|g\rangle+\psi_{e}\left(\mathbf{r}_{a}, t\right)|e\rangle
\end{aligned}
$$

These components satisfy the multicomponent GrossPitaevskii (GP) equations

$$
\begin{aligned}
i \hbar \frac{\partial \psi_{g}}{\partial t} & =H_{0}^{(g)} \psi_{g}\left(\mathbf{r}_{a}, t\right)+E_{g} \psi_{g}\left(\mathbf{r}_{a}, t\right) \\
& +2 \hbar \Omega_{0} \cos \left(\mathbf{k}_{L} \cdot \mathbf{r}_{a}\right) f(t) \cos \left(\omega_{L} t\right) \psi_{e}\left(\mathbf{r}_{a}, t\right) \\
& +g N\left(\left|\psi_{g}\left(\mathbf{r}_{a}, t\right)\right|^{2}+\left|\psi_{e}\left(\mathbf{r}_{a}, t\right)\right|^{2}\right) \psi_{g}\left(\mathbf{r}_{a}, t\right)
\end{aligned}
$$

and

$$
\begin{aligned}
i \hbar \frac{\partial \psi_{e}}{\partial t} & =H_{0}^{(e)} \psi_{e}\left(\mathbf{r}_{a}, t\right)+E_{e} \psi_{e}\left(\mathbf{r}_{a}, t\right) \\
& +2 \hbar \Omega_{0} \cos \left(\mathbf{k}_{L} \cdot \mathbf{r}_{a}\right) f(t) \cos \left(\omega_{L} t\right) \psi_{g}\left(\mathbf{r}_{a}, t\right) \\
& +g N\left(\left|\psi_{g}\left(\mathbf{r}_{a}, t\right)\right|^{2}+\left|\psi_{e}\left(\mathbf{r}_{a}, t\right)\right|^{2}\right) \psi_{e}\left(\mathbf{r}_{a}, t\right)
\end{aligned}
$$

The solution of these equations, over the time of the laser pulse can be approximated if (1) the pulse time is short enough so that $\delta t_{1} \ll \hbar / \mu$ where $\mu$ is the condensate chemical potential, and (2) if the single-photon Rabi frequency is small compared to the detuning from resonance, i.e., $\Omega_{0} \ll \Delta$. The detuning is defined by $\hbar \Delta=E_{e}-E_{g}-\hbar \omega_{L}$. We will see that the solution is valid even for a strong pulse.

By approximately solving the multi-component GP equations over the duration of the pulse, the details of which are given in Appendix $\mathrm{A}$, the effect of a short-time, 
strong-field, standing-wave laser pulse on the condensate wave function is given by

$$
\begin{aligned}
\phi_{g}\left(\mathbf{r}_{a}, t_{1+}\right) \approx & e^{i \Omega_{2} \delta t_{1}} \\
& \times e^{i \Omega_{2} \delta t_{1} \cos \left(2 \mathbf{k}_{L} \cdot \mathbf{r}_{a}\right)} \phi_{g}\left(\mathbf{r}_{a}, t_{1-}\right) .
\end{aligned}
$$

where

$$
\Omega_{2} \equiv \frac{\Omega_{0}^{2}}{2 \Delta}
$$

is the two-photon Rabi frequency.

It is possible to represent the effect of the pulse in momentum space by using the Bessel generating function [23].

$$
e^{\frac{1}{2} z\left(t-\frac{1}{t}\right)}=\sum_{n=-\infty}^{\infty} t^{n} J_{n}(z)
$$

Letting $t=i e^{2 i \mathbf{k}_{L} \cdot \mathbf{r}_{a}}$ and $z=\Omega_{2} \delta t_{1}$ gives

$$
e^{i \Omega_{2} \delta t_{1} \cos \left(2 \mathbf{k}_{L} \cdot \mathbf{r}_{a}\right)}=\sum_{n=-\infty}^{\infty} i^{n} J_{n}\left(\Omega_{2} \delta t_{1}\right) e^{2 n i \mathbf{k}_{L} \cdot \mathbf{r}_{a}} .
$$

Hence, the wave function just after the pulse can be written as

$$
\begin{aligned}
\phi_{g}\left(\mathbf{r}_{a}, t_{1+}\right) & \approx e^{i \Omega_{2} \delta t_{1}} \\
& \times \sum_{n=-\infty}^{\infty} i^{n} J_{n}\left(\Omega_{2} \delta t_{1}\right) e^{2 n i \mathbf{k}_{L} \cdot \mathbf{r}_{a}} \phi_{g}\left(\mathbf{r}_{a}, t_{1-}\right)
\end{aligned}
$$

The $e^{2 n i \mathbf{k}_{L} \cdot \mathbf{r}_{a}} \phi_{g}\left(\mathbf{r}_{a}, t_{1-}\right)$ factor in the $n^{\text {th }}$ term in the above sum is the wave function of the original zeromomentum condensate kicked into a momentum state centered at $\mathbf{p}_{n}=2 n \hbar \mathbf{k}_{L}$ and expressed in the positionspace representation. Thus the amplitude for a condensate atom starting from zero momentum and kicked into momentum state $2 n \hbar \mathbf{k}_{L}$ by the pulse is $i^{n} J_{n}\left(\Omega_{2} \delta t_{1}\right)$. We now turn to the evolution of the condensate wave function between pulses.

\section{B. Evolution between pulses}

Between the first laser pulse at $t=t_{1}$ and the second at $t=t_{2}$, the evolution of an atom in momentum state $\mathbf{p}_{n}=2 n \hbar \mathbf{k}_{L}$ can be approximated as a free particle under conditions described below. The phase of such an atom thus evolves as $e^{-i E_{n}\left(t_{2}-t_{1}\right) / \hbar}$ where $E_{n}=p_{n}^{2} / 2 m$ is its kinetic energy and the atom moves with velocity $\mathbf{v}_{n}=$ $2 n \hbar \mathbf{k}_{L} / m$. For non-zero momentum states, as long as the number of atoms outcoupled into them is too small to be detected experimentally or the time scale over which they evolve is small compared to $\hbar / \mu$, mean-field effects can be ignored. Thus we can write the condensate wave function during the time $t_{1}<t<t_{2}$ as

$$
\begin{aligned}
\phi_{g}\left(\mathbf{r}_{a}, t\right) & =e^{i \Omega_{2} \delta t_{1}} \sum_{n=-\infty}^{\infty} i^{n} J_{n}\left(\Omega_{2} \delta t\right) e^{-i E_{n}\left(t-t_{1}\right) / \hbar} \\
& \times e^{2 n i \mathbf{k}_{L} \cdot \mathbf{r}_{a}} \phi_{g}\left(\mathbf{r}_{a}-\mathbf{v}_{n}\left(t-t_{1}\right), t_{1}\right)
\end{aligned}
$$

It is clear that, although the above equation implies that there is a finite probability for atoms to be outcoupled into a momentum state that is any multiple of $2 \hbar \mathbf{k}_{L}$, above some maximum order, $n_{\max }$, there will be too few atoms present to be detected experimentally. Here we will assume that the Raman-Nath approximation holds, that is, the only momentum orders appreciably populated are ones in which the atoms moved only a small fraction of their de Broglie wavelengths during the time between pulses. In this case,

$$
\begin{aligned}
\phi_{g}\left(\mathbf{r}_{a}, t\right) & \approx e^{i \Omega_{2} \delta t_{1}} \sum_{n=-n_{\max }}^{n_{\max }} i^{n} J_{n}\left(\Omega_{2} \delta t_{1}\right) e^{-i E_{n}\left(t-t_{1}\right) / \hbar} \\
& \times e^{2 n i \mathbf{k}_{L} \cdot \mathbf{r}_{a}} \phi_{g}\left(\mathbf{r}_{a}, t_{1}\right) .
\end{aligned}
$$

Note that we have neglected the motion of the non-zero momentum orders over the time interval $t-t_{1}$. We shall assume that the Raman-Nath approximation holds hereafter. Later, when we consider the $N$-pulse case, we will assume that this holds for the entire pulse sequence. Next we analyze the effect of the second pulse.

\section{Effect of the second pulse}

The effect of the second pulse can be described by applying the exponential in Eq. (9) to the wave function in the Eq. (15). The wave function just after the application of the second pulse is

$$
\begin{aligned}
\phi_{g}\left(\mathbf{r}_{a}, t_{2+}\right) & \approx e^{i \Omega_{2}\left(\delta t_{1}+\delta t_{2}\right)} \\
& \times \sum_{n=-n_{\max }}^{n_{\max }} i^{n} J_{n}\left(\Omega_{2} \delta t_{1}\right) e^{-i E_{n}\left(t_{2}-t_{1}\right) / \hbar} \\
& \times e^{2 n i \mathbf{k}_{L} \cdot \mathbf{r}_{a}} e^{i \Omega_{2} \delta t_{2} \cos \left(2 \mathbf{k}_{L} \cdot \mathbf{r}_{a}\right)} \phi_{g}\left(\mathbf{r}_{a}, t_{1}\right) .
\end{aligned}
$$

The exponential describing the effect of the second pulse can also be expanded in a truncated series of Bessel functions and we have.

$$
\begin{aligned}
\phi_{g}\left(\mathbf{r}_{a}, t_{2+}\right) & \approx e^{i \Omega_{2}\left(\delta t_{1}+\delta t_{2}\right)} \\
& \times \sum_{n=-n_{\max }}^{n_{\max }} \sum_{n^{\prime}=-n_{\max }}^{n_{\max }} i^{n+n^{\prime}} J_{n}\left(\Omega_{2} \delta t_{1}\right) \\
& \times J_{n^{\prime}}\left(\Omega_{2} \delta t_{2}\right) e^{-i E_{n}\left(t_{2}-t_{1}\right) / \hbar} e^{2\left(n+n^{\prime}\right) i \mathbf{k}_{L} \cdot \mathbf{r}_{a}} \\
& \times \phi_{g}\left(\mathbf{r}_{a}, t_{1}\right) .
\end{aligned}
$$

Changing summation indexes as $m \equiv n+n^{\prime}$ we can write

$$
\phi_{g}\left(\mathbf{r}_{a}, t_{2+}\right) \approx e^{i \Omega_{2}\left(\delta t_{1}+\delta t_{2}\right)}
$$




$$
\times \sum_{m=-2 n_{\max }}^{2 n_{\max }} A_{m} e^{2 m i \mathbf{k}_{L} \cdot \mathbf{r}_{a}} \phi_{g}\left(\mathbf{r}_{a}, t_{1}\right)
$$

where

$$
A_{m} \equiv i^{m} \sum_{n=-\infty}^{\infty} J_{m-n}\left(\Omega_{2} \delta t_{2}\right) J_{n}\left(\Omega_{2} \delta t_{1}\right) e^{-i E_{n}\left(t_{2}-t_{1}\right) / \hbar}
$$

Note that we have extended the limits of the summation back to infinity. This is possible because, in the RamanNath regime, the values of $J_{n}\left(\Omega_{2} \delta t\right)$ for $n>n_{\max }$ are sufficiently small that we incur little error in including these extra terms. So, finally, we have

$$
\begin{aligned}
\phi_{g}\left(\mathbf{r}_{a}, t\right) & \approx e^{i \Omega_{2}\left(\delta t_{2}+\delta t_{1}\right)} \\
& \times \sum_{m=-\infty}^{\infty} A_{m} e^{2 m i \mathbf{k}_{L} \cdot \mathbf{r}_{a}} \phi_{g}\left(\mathbf{r}_{a}, t_{1}\right)
\end{aligned}
$$

The quantity $A_{m}$ is the probability amplitude for an atom to be in momentum state $\mathbf{p}_{m}=2 m \hbar \mathbf{k}_{L}$.

\section{GENERAL FEATURES OF THE SOLUTION FOR TWO PULSES}

Before deriving some general features of the two-pulse amplitude, it will be convenient to rewrite Eq. (19) in a form where the time interval is measured in appropriate units. To this end we define the period, $T_{T}$, of free oscillation of a free particle whose momentum is $\mathbf{p}_{1}=2 \hbar \mathbf{k}_{L}$ such that:

$$
\left(\frac{p_{1}^{2}}{2 m}\right) T_{T} / \hbar=2 \pi
$$

This is the Talbot time defined above. In this case

$$
\begin{aligned}
\frac{E_{n}\left(t_{2}-t_{1}\right)}{\hbar} & =n^{2}\left(\frac{p_{1}^{2}}{2 m}\right) \frac{\left(t_{2}-t_{1}\right)}{\hbar} \\
& =\frac{2 \pi n^{2}\left(t_{2}-t_{1}\right)}{T_{T}} \equiv 2 \pi \beta_{1} n^{2},
\end{aligned}
$$

where we have introduced the time interval parameter as $\beta_{1} \equiv\left(t_{2}-t_{1}\right) / T_{T}$, that is, the time between pulses measured in units of the Talbot time. Also defining the pulse area parameters as $\alpha_{1} \equiv \Omega_{2} \delta t_{1}$ and $\alpha_{2} \equiv \Omega_{2} \delta t_{2}$, we can write $A_{m}$ as

$$
A_{m}\left(\alpha_{1}, \alpha_{2}, \beta_{1}\right)=i^{m} \sum_{n=-\infty}^{\infty} J_{m-n}\left(\alpha_{2}\right) e^{-2 \pi i n^{2} \beta_{1}} J_{n}\left(\alpha_{1}\right)
$$

We will use this form of the two-pulse amplitude to demonstrate some its general features.

\section{A. Symmetry of the momentum distribution}

There are two general symmetries exhibited by the expression in Eq. (23). The first symmetry is that the amplitudes for opposite momentum orders are equal for a given pulse sequence, that is

$$
A_{m}\left(\alpha_{1}, \alpha_{2}, \beta_{1}\right)=A_{-m}\left(\alpha_{1}, \alpha_{2}, \beta_{1}\right) .
$$

To show this symmetry, we write the expression for $A_{-m}\left(\alpha_{1}, \alpha_{2}, \beta_{1}\right)$ which is

$$
\begin{aligned}
A_{-m}\left(\alpha_{1}, \alpha_{2}, \beta_{1}\right) & =i^{-m} \sum_{n=-\infty}^{\infty} J_{-m-n}\left(\alpha_{2}\right) \\
& \times e^{-2 \pi i n^{2} \beta_{1}} J_{n}\left(\alpha_{1}\right) .
\end{aligned}
$$

Using the Bessel generating function [23], it is easy to show that $J_{-n}(z)=(-)^{n} J_{n}(z)$ so that

$$
\begin{aligned}
A_{-m}\left(\alpha_{1}, \alpha_{2}, \beta_{1}\right) & =i^{-m} \sum_{n=-\infty}^{\infty}(-)^{m+n} J_{m+n}\left(\alpha_{2}\right) \\
& \times e^{-2 \pi i n^{2} \beta_{1}}(-)^{n} J_{-n}\left(\alpha_{1}\right) \\
& =i^{m} \sum_{n=-\infty}^{\infty} J_{m+n}\left(\alpha_{2}\right) \\
& \times e^{-2 \pi i n^{2} \beta_{1}} J_{-n}\left(\alpha_{1}\right) .
\end{aligned}
$$

Changing the summation index to $n^{\prime}=-n$ we have

$$
\begin{aligned}
A_{-m}\left(\alpha_{1}, \alpha_{2}, \beta_{1}\right) & =i^{m} \sum_{n^{\prime}=-\infty}^{\infty} J_{m-n^{\prime}}\left(\alpha_{2}\right) \\
& \times e^{-2 \pi i\left(-n^{\prime}\right)^{2} \beta_{1}} J_{n^{\prime}}\left(\alpha_{1}\right),
\end{aligned}
$$

and so

$$
A_{-m}\left(\alpha_{1}, \alpha_{2}, \beta_{1}\right)=A_{m}\left(\alpha_{1}, \alpha_{2}, \beta_{1}\right) .
$$

Thus all distributions are exactly symmetric with respect to momentum order under these conditions. Physically when atoms make a transition to a non-zero momentum state they must absorb photons from one laser beam and emit into the other beam. Equation (26) holds because, for standing-wave laser beams, there is no preference for choosing which beam photons are absorbed and into which emitted. Absorbing from opposite beams results in populating opposite final momentum states.

The second symmetry is that the amplitude for a given pair of pulses separated by an interval $\beta_{1}$ is the complex conjugate of the amplitude for an interval of $1-\beta_{1}$ for fixed $\alpha_{1}$ and $\alpha_{2}$. Thus we have

$$
A_{m}\left(\alpha_{1}, \alpha_{2}, 1-\beta_{1}\right)=A_{m}^{*}\left(\alpha_{1}, \alpha_{2}, \beta_{1}\right) \quad \beta_{1} \leq 1 / 2 .
$$

If we only consider interval times $\beta_{1} \leq 1 / 2$, it is easy to see that this holds in Eq. (19). Since $e^{-2 \pi i n^{2}\left(1-\beta_{1}\right)}=$ $e^{2 \pi i n^{2} \beta_{1}}$ gives the complex conjugate of the exponential 
(a)

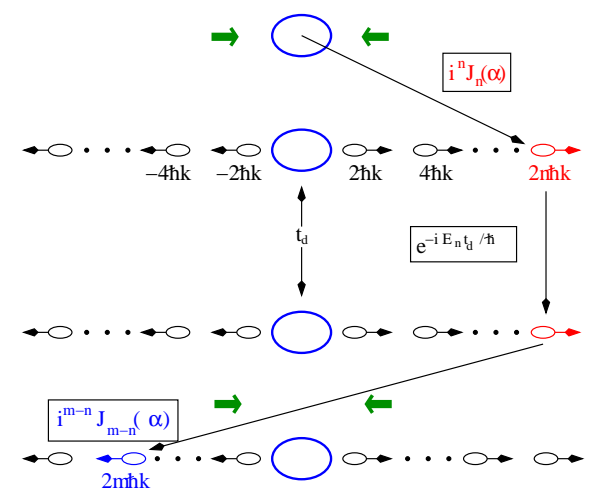

(b)

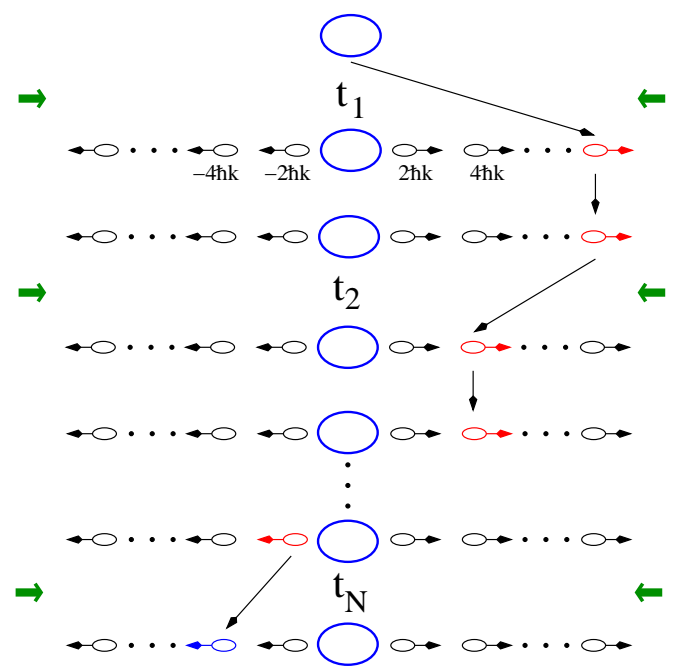

FIG. 1: (a) (color online) The final distribution of the condensate atoms over the momentum states after the application of two standing-wave pulses separated by an interval can be understood in terms of interfering quantum pathways. The amplitude for the system to jump from the initial zero-momentum state at the top to the final state at the bottom along the path shown is the product of the amplitudes (shown in boxes) for the three legs that compose the path. The total amplitude is coherent summation of this composite single-path amplitude over all possible paths. (b) The general case of $N$ pulses is illustrated in the right panel. We assume that pulse 1 is applied at $t_{1}$, pulse 2 at $t_{2}$, etc.

in the $\beta_{1}$ amplitude. Given the fact that the Bessel functions are all real, conjugating the exponential conjugates the entire expression. Equation (27) also implies that the probability distribution for two-pulse sequences with intervals $\beta_{1}$ and $1-\beta_{1}$ are identical. This result will be useful later to constrain the parameter space in the momentum space design procedure described below.

\section{B. Special values of the time interval}

Two important results can be obtained for the quantity $A_{m}\left(\alpha_{1}, \alpha_{2}, \beta_{1}\right)$ when the interval time has special values. The two special cases are (1) $\beta_{1}=\frac{1}{2}$, and (2) $\beta_{1}=1$. We consider each of these in turn.

The first case occurs when the time interval between standing-wave pulses equals half of the Talbot time. Here, the exponential factor in Eq. (23) becomes

$$
e^{-2 \pi i n^{2} \beta_{1}}=e^{-\pi i n^{2}}=(-)^{n^{2}}=(-)^{n} .
$$

The last equality can be seen by noting that the square of an even integer is even and the square of an odd integer is odd. Thus, the probability amplitude becomes

$$
A_{m}\left(\alpha_{1}, \alpha_{2}, \frac{1}{2}\right)=i^{m} \sum_{n=-\infty}^{\infty}(-)^{n} J_{m-n}\left(\alpha_{2}\right) J_{n}\left(\alpha_{1}\right)
$$

This expression can be summed exactly as follows. We write again the Bessel generating function

$$
\begin{aligned}
e^{\frac{1}{2} \alpha_{1}\left(t-\frac{1}{t}\right)} & =\sum_{n=-\infty}^{\infty} t^{n} J_{n}\left(\alpha_{1}\right) \\
e^{-\frac{1}{2} \alpha_{2}\left(t-\frac{1}{t}\right)} & =\sum_{n^{\prime}=-\infty}^{\infty}(-)^{n^{\prime}} t^{n^{\prime}} J_{n^{\prime}}\left(\alpha_{2}\right) .
\end{aligned}
$$

In the second equality, we have let $\alpha_{1} \rightarrow-\alpha_{2}$ and used the identity $J_{n}(-\alpha)=(-)^{n} J_{n}(\alpha)$. Equating the product of the right-hand-sides with the product of the left-hand-side of the above two equations and letting $m=n+n^{\prime}$ yields the following

$$
\begin{aligned}
e^{-\frac{1}{2}\left(\alpha_{2}-\alpha_{1}\right)\left(t-\frac{1}{t}\right)} & =\sum_{m=-\infty}^{\infty} i^{m} A_{m}\left(\alpha_{1}, \alpha_{2}, \frac{1}{2}\right) t^{m} \\
& =\sum_{m=-\infty}^{\infty}(-)^{m} J_{m}\left(\alpha_{2}-\alpha_{1}\right) t^{m}
\end{aligned}
$$

where the second equality comes from a direct application of the Bessel generating function to the exponential on the left-hand-side. Since the equality of the two sums must hold for any value of $t$, the coefficients of $t^{m}$ in the sums must be equal. And so,

$$
A_{m}\left(\alpha_{1}, \alpha_{2}, \frac{1}{2}\right)=i^{m} J_{m}\left(\alpha_{2}-\alpha_{1}\right)
$$

One consequence of this is that, for equal-area pulses and when the interval between pulses is one-half of the Talbot 


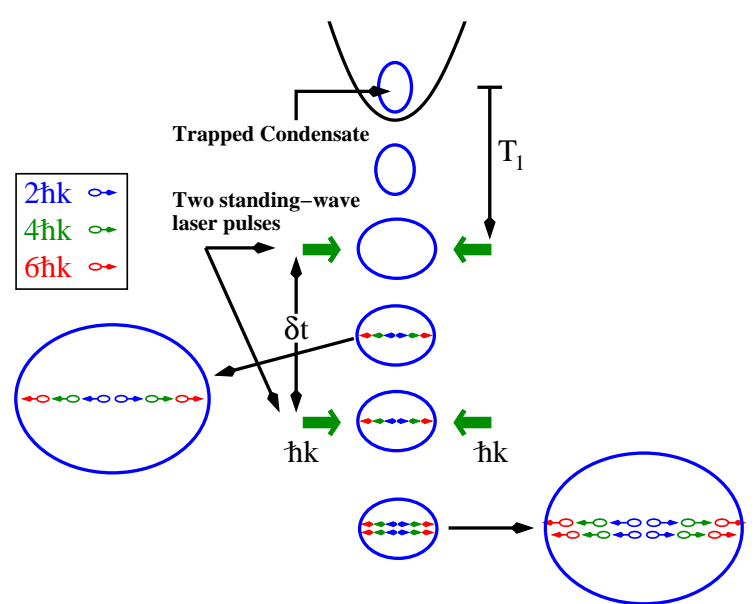

(a)

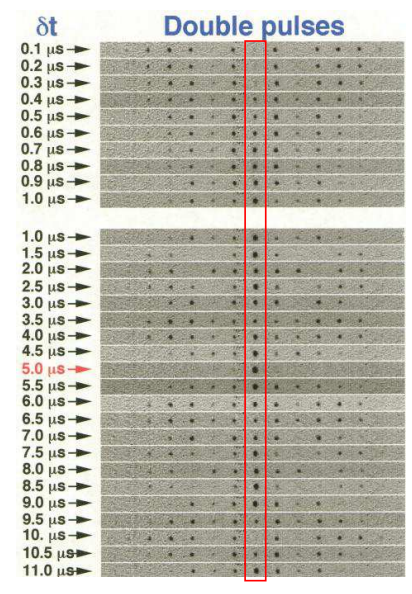

(b)

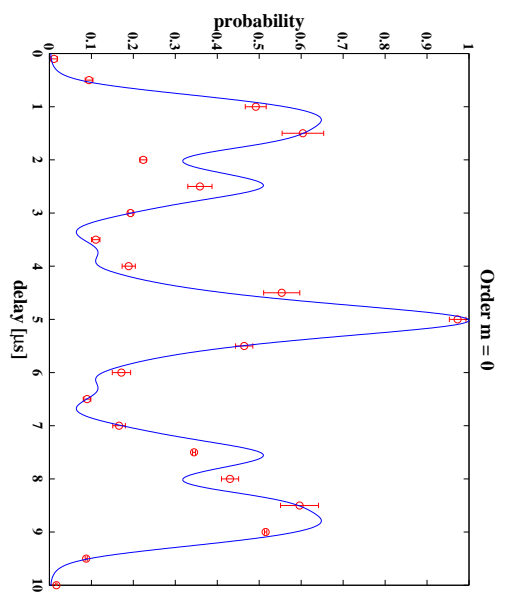

(c)

FIG. 2: (color online) Comparison of the theory presented in the text with the results of the temporal Talbot experiment reported in Ref. [11]. (a) The left panel shows the steps of this experiment. Two $100-\mathrm{ns}, 1 \mathrm{~W} / \mathrm{cm}^{2}$, standing-wave laser pulses were applied to a ${ }^{23} \mathrm{Na}$ BEC after allowing the condensate to expand for $1.2 \mathrm{~ms}$. The pulses were separated in time by an interval, $\delta t$, which varied between 0.1 and $11 \mu \mathrm{s}$. (b) The middle panel shows the images taken after allowing the condensate to expand for a further $6.2 \mathrm{~ms}$. Each column of dots corresponds to a specific final momentum state. The highlighted column corresponds to zero momentum while the column just to the right (left) of it corresponds to the $2 \hbar k$ ( $-2 \hbar k$ ) momentum state. (c) The right panel shows a comparison of the prediction of Eq. (19) with the normalized pixel count of the dots of the highlighted column.

time, (that is, when $\alpha_{1}=\alpha_{2}$ and $\beta_{1}=1 / 2$ ) the amplitudes for all non-zero momentum states are zero and the condensate is unchanged. This effect was verified experimentally and reported in Ref. [1]. Figure 2(b) shows this effect where a double-pulse delay of $5 \mu$ s results in no change to the original condensate.

In the second case where $\beta_{1}=1$ the exponential in the expression for $A_{m}\left(\alpha_{1}, \alpha_{2}, 1\right)$ equals unity for all values of the summation index $n$. Thus we can write

$$
\begin{aligned}
A_{m}\left(\alpha_{1}, \alpha_{2}, 1\right) & =i^{m} \sum_{n=-\infty}^{\infty} J_{m-n}\left(\alpha_{2}\right) J_{n}\left(\alpha_{1}\right) \\
& =i^{m} J_{m}\left(\alpha_{2}+\alpha_{1}\right)
\end{aligned}
$$

where the second equality is derived by a method similar to that which produced Eq. (32). This result suggests that two standing-wave pulses separated in time by one Talbot time have the same effect as a single pulse whose area is the sum of the areas of the two pulses.

\section{Physical interpretation of the probability amplitude}

The general formula for the probability amplitude, Eq. (19), can be understood as the superposition of amplitudes of multiple pathways from the given initial to the given final state. One such pathway is illustrated in Fig. [1(a). This figure depicts a particular quantum pathway from the fixed initial zero-momentum state to a fixed final state whose momentum is $p_{m}=2 m \hbar k$. The amplitude for an atom to go between these states via the path shown is the product of the amplitudes for the three legs of the path. The first leg is a momentum jump, caused by the first pulse, from the zero-momentum state to the momentum state $p=p_{n}=2 n \hbar k$ with amplitude $i^{n} J_{n}(\alpha)$. In the second leg of the path, atoms in the momentum state $p=p_{n}$, whose energy is $E_{n}=p_{n}^{2} / 2 m$, evolve as free particles during the short-time interval between pulses. Thus the amplitude to "jump" from the time just after the first pulse to just before the second pulse is $e^{-i E_{n} t_{d} / \hbar}$. The final leg of the path shown is another momentum jump, caused by the second pulse, from $p=p_{n}$ to $p=p_{m}$ and whose amplitude is $i^{m-n} J_{m-n}(\alpha)$. The amplitude to proceed from the initial to the final state is the product of the amplitudes for the three legs.

This pathway proceeds from the initial state to the final state via the momentum state $p_{n}$. The system can make the transition between these initial and final states via any state $p_{n}$ and since these different pathways are not detected, the total amplitude for the system to jump from the initial to the final state is the coherent summation of these individual amplitudes given in Eq. (19). This quantum pathways interpretation will enable an easy generalization of the two-pulse amplitude to the $N$-pulse case. 


\section{Comparison with experiment}

The validity of this theory for two pulses was tested in an experiment and reported in Ref. ([1] ). In this experiment, a BEC consisting of $3 \times 10^{6} \mathrm{Na}$ atoms confined in the $F=1, m_{F}=-1$ ground state by a timeaveraged orbiting potential (TOP) trap 24] were released and allowed to expand for $1.2 \mathrm{~ms}$ as illustrated in the left panel of Fig. (2). Next, two 589-nm-wavelength, 100-nsduration, standing-wave, linearly polarized laser pulses were applied with a time interval between them which varied between 1 and $10 \mu \mathrm{s}$. The intensity of the pulses was about $1 \mathrm{~W} / \mathrm{cm}^{2}$ and they were detuned by approximately $600 \mathrm{MHz}$ from the $3 S_{1 / 2}, F=1 \rightarrow 3 P_{3 / 2}, F^{\prime}=2$ transition. The condensate was then allowed to expand for a further $6.2 \mathrm{~ms}$ at which time an absorption image was taken. This last expansion enabled atoms in nonzero momentum states to leave the condensate and the resulting image is a measurement of the momentum-space distribution immediately after the second laser pulse.

The middle panel in Fig. (2) shows the results of these absorption images for varying interval times between the pulses. Each row shows a picture of the data for a particular interval time while the columns indicate particular momentum states. The center column highlighted shows atoms in the zero-momentum state. The graph shown on the right-hand panel is a comparison of the normalized pixel counts of the dots in the zero-momentum states (highlighted column in panel (b)) with the theory curve $\left|A_{0}\left(\alpha_{0}, \alpha_{0}, \beta\right)\right|^{2}$ plotted as a function of $\beta$ and where $\alpha_{0}$ is the product of the two-photon Rabi frequency and the pulse time corresponding to the experimental conditions. It is important to note that there are no adjustable parameters in this calculation. One can see that there is good agreement between theory and experiment which in turn lends support for the model presented above.

\section{N-PULSE THEORY}

Consider a condensate that is subjected to a sequence of $N$ pulses with arbitrary pulse areas and times. As shown in Fig. 1(b), we assume that pulse 1 is applied at $t=t_{1}$ and has pulse area $\alpha_{1}$, pulse 2 at $t=t_{2}$ with area $\alpha_{2}, \ldots$, pulse $N$ at $t=t_{N}$ with area $\alpha_{N}$. If we label the momentum state jumped to at the time of pulse $k$ as $n_{k}$, where $0 \leq k \leq N$, then a single quantum pathway through the entire $N$-pulse sequence can be labeled by specifying the index of the momentum state the system jumps to after each pulse: $\left(n_{1}, n_{2}, \ldots, n_{N-1}\right)$. Then it is easy to write the amplitude for a particular $N$-pulse pathway by analogy with the two-pulse case. The resulting amplitude for a single quantum pathway whose starting momentum state is $2 n_{0} \hbar k$ and whose ending state is $2 n_{N} \hbar k$ for an $N$-pulse sequence labeled in this way is

$$
\begin{aligned}
A_{n_{N}, n_{0}}^{(N)}(\boldsymbol{\alpha}, \boldsymbol{\delta} t, \boldsymbol{n}) & =\left(i^{n_{1}-n_{0}} J_{n_{1}-n_{0}}\left(\alpha_{1}\right) e^{-i E_{n_{1}}\left(t_{2}-t_{1}\right) / \hbar}\right) \\
& \times\left(i^{n_{2}-n_{1}} J_{n_{2}-n_{1}}\left(\alpha_{2}\right) e^{-i E_{n_{2}}\left(t_{3}-t_{2}\right) / \hbar}\right)
\end{aligned}
$$

$$
\times \cdots\left(i^{n_{N}-n_{N-1}} J_{n_{N}-n_{N-1}}\left(\alpha_{N}\right)\right),
$$

where we have assumed that the initial momentum state is not zero but rather labeled by $n_{0}$ and we have not considered any interval following the final pulse at $t=t_{N}$.

The vectors $\boldsymbol{\alpha}$ and $\boldsymbol{\delta} t$ label the areas and intervals of the applied pulse sequence.

$$
\begin{aligned}
\boldsymbol{\alpha} & \equiv\left(\alpha_{1}, \alpha_{2}, \ldots, \alpha_{N}\right) \\
\boldsymbol{\delta} t & \equiv\left(\delta t_{1}=t_{2}-t_{1}, \ldots, \delta t_{N-1}=t_{N}-t_{N-1}\right) .
\end{aligned}
$$

It will be convenient to rescale the intervals in units of the Talbot time as was done for two pulses. Thus we define $\beta_{k}=\delta_{k} / T_{T}$ for $1 \leq k \leq N-1$. Hence we can rewrite Eq. (34) as

$$
\begin{aligned}
A_{n_{N}, n_{0}}^{\left(n_{1}, \ldots, n_{N-1}\right)}(\boldsymbol{\alpha}, \boldsymbol{\beta}) & =i^{n_{N}-n_{0}} J_{n_{1}-n_{0}}\left(\alpha_{1}\right) e^{-2 \pi i n_{1}^{2} \beta_{1}} \\
& \times J_{n_{2}-n_{1}}\left(\alpha_{2}\right) e^{-2 \pi i n_{2}^{2} \beta_{2}} \ldots \\
& \times e^{-2 \pi i n_{N-1}^{2} \beta_{N-1}} J_{n_{N}-n_{N-1}}\left(\alpha_{N}\right),
\end{aligned}
$$

To get the full amplitude to jump from the initial momentum state, labeled by $n_{0}$, to the final momentum state, labeled by $n_{N}$, we coherently sum over all of the single-path amplitudes. This yields the following.

$$
\begin{aligned}
A_{n_{N}, n_{0}}^{(N)}(\boldsymbol{\alpha}, \boldsymbol{\beta}) & =\sum_{n_{1}} \cdots \sum_{n_{N-1}} A_{n_{N}, n_{0}}^{\left(n_{1}, \ldots, n_{N-1}\right)}(\boldsymbol{\alpha}, \boldsymbol{\beta}) \\
& =i^{n_{N}-n_{0}} \sum_{n_{1}} \cdots \sum_{n_{N-1}} J_{n_{1}-n_{0}}\left(\alpha_{1}\right) \\
& \times e^{-2 \pi i n_{1}^{2} \beta_{1}} J_{n_{2}-n_{1}}\left(\alpha_{2}\right) e^{-2 \pi i n_{2}^{2} \beta_{2}} \\
& \cdots e^{-2 \pi i n_{N-1}^{2} \beta_{N-1}} J_{n_{N}-n_{N-1}}\left(\alpha_{N}\right) .
\end{aligned}
$$

This is the general result for the amplitude to jump from an initial momentum state of $p_{i}=2 n_{0} \hbar k$ to a final momentum state of $p_{f}=2 n_{N} \hbar k$ due to the application of $N$ pulses whose areas are $\alpha_{1}, \ldots, \alpha_{N}$ separated by $N-1$ intervals of durations (expressed in Talbot-time units) $\beta_{1}, \ldots, \beta_{N-1}$. It holds as long as the Raman-Nath approximation is valid for all pulses, i.e., that atoms in non-zero-order momentum states do not move an appreciable distance compared to the condensate size during the entire pulse sequence. We can now use this result to design sequences of such pulses and intervals to engineer a specified momentum-state probability distribution.

\section{ENGINEERING MOMENTUM-STATE PROBABILITY DISTRIBUTIONS}

\section{A. Least-squares design}

Designing a pulse sequence to engineer a specified probability distribution across the momentum states $2 m \hbar k$ is straightforward. First, the momentum distribution is described by specifying the set of desired probabilities $\left\{p_{m}\right\}$ for momentum orders $2 m \hbar k$ for all $m$. We will 
(a)
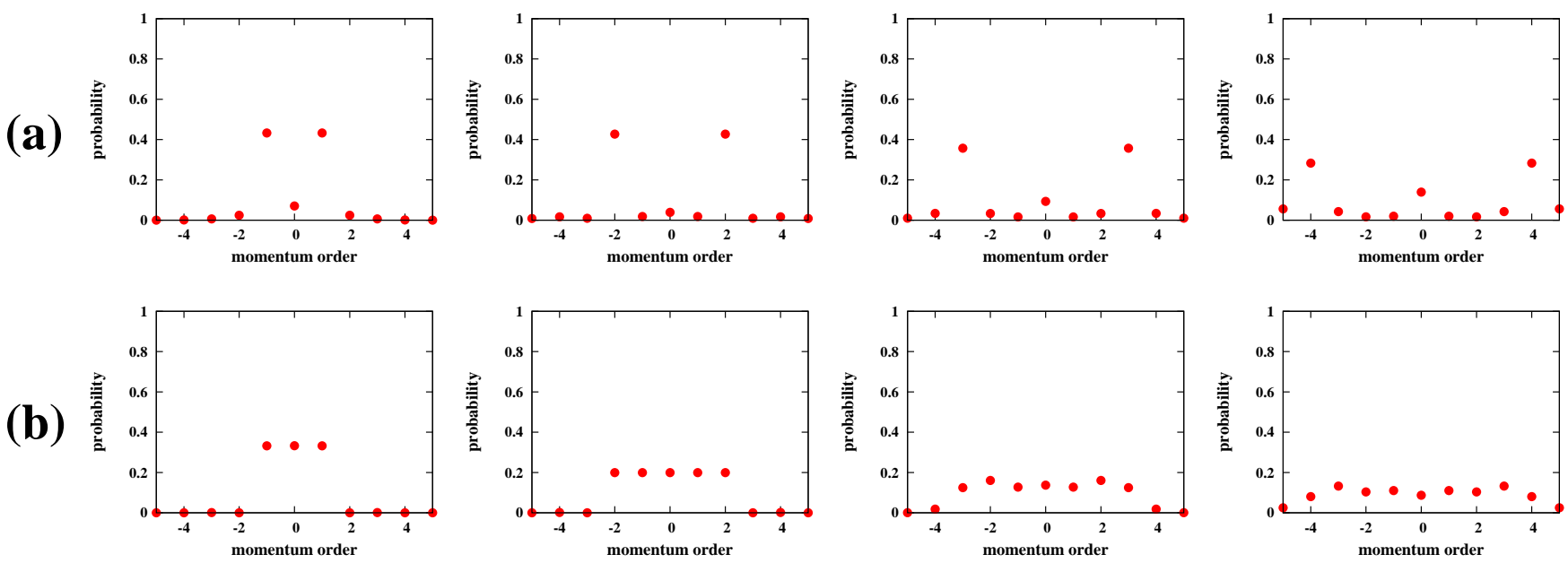

FIG. 3: (color online) The figure above shows examples of shaping momentum distributions using only two pulses. The shaping parameters are the pulse areas, $\alpha_{1}$ and $\alpha_{2}$, and the interval between them, $\beta_{1}$. All of the plots above show the probabilities of atoms being in momentum orders $2 m \hbar k$ where $-5 \leq m \leq 5$. Each plot is annotated with the values of $\alpha_{1}$, $\beta_{1}$, and $\alpha_{2}$ that produced each momentum distribution. (a) The four plots in the top panel exhibit (from left to right) the distributions $D_{\text {mag }}^{(2)}(m)$ where the two momentum states $-2 m \hbar k$ and $+2 m \hbar k$ are equally populated and where $1 \leq m \leq 4$. (b) The four plots in the bottom panel depict (again from left to right) distributions $D_{\text {range }}^{(2)}(m)$ where all of the states in the range between $-2 m \hbar k$ and $+2 m \hbar k$ are equally populated and where $1 \leq m \leq 4$.

refer to this set of numbers as the momentum probability distribution. This set of numbers must satisfy several conditions to be a valid momentum probability distribution. Each $p_{m}$ must be a probability and the distribution must be normalized so that

$$
0 \leq p_{m} \leq 1, \quad-\infty<m<\infty,
$$

and

$$
\sum_{m=-\infty}^{\infty} p_{m}=1
$$

Furthermore, since all of the pulses are assumed to be standing waves, by symmetry the probability for $2 m \hbar k$ must equal that for $-2 m \hbar k$ and thus

$$
p_{m}=p_{-m}, \quad-\infty<m<\infty .
$$

Once the momentum probability distribution is specified, the least squares procedure can be carried out by defining the $N$-pulse, least-squares cost function:

$$
F_{L S}^{(N)}(\boldsymbol{\alpha}, \boldsymbol{\beta}) \equiv \sum_{m=-\infty}^{\infty}\left[p_{m}-\left|A_{m, 0}^{(N)}(\boldsymbol{\alpha}, \boldsymbol{\beta})\right|^{2}\right]^{2}
$$

and finding the values of the parameters $\boldsymbol{\alpha}=\boldsymbol{\alpha}^{\text {min }}$ and $\boldsymbol{\beta}=\boldsymbol{\beta}^{\text {min }}$ that produces the global minimum of $F_{L S}(\boldsymbol{\alpha}, \boldsymbol{\beta})$.

In general, this minimization must be done numerically. It is also subject to certain constraints which both derive from the Raman-Nath approximation. First, we assume that all intervals are less than or equal to one Talbot time, or $0 \leq \beta_{n} \leq 1$ for $1 \leq n \leq N$. Second, there is a limit on the size of each individual pulse area because too large an area will produce population in such a high momentum state that there will be appreciable motion during the pulse sequence. Thus there is a maximum value, $\alpha_{\max }$, such that $0 \leq \alpha_{n} \leq \alpha_{\max }$. These constraints also limit the total number of pulses that can be practically applied while still satisfying all of the conditions described above. However, as we shall see, many important momentum probability distributions can be achieved with high fidelity using only two or three pulses.

We implemented the least-squares minimization procedure described above to design two-pulse and threepulse sequences to produce momentum distributions in two categories. The first category is a distribution where all of the population appears in the two momentum states where the magnitude is $2 n \hbar k$ (that is, $+2 n \hbar k$ or $-2 n \hbar k$ ) or equivalently where $p_{n}=p_{-n}=1 / 2$. We will use the label, $D_{m a g}^{(N)}(n)$, to stand for the distribution determined from the $N$-pulse, least-squares procedure when the desired distribution is of this type. The other kind of distribution we considered has equal populations in the range of momentum states between $\pm 2 n \hbar k$. In this case $p_{-n}=\ldots=p_{n}=1 /(2 n+1)$. The distribution produced by the $N$-pulse, least-squares procedure when this type of distribution will be labeled by $D_{\text {range }}^{(N)}(n)$.

These categories of distributions are interesting because they act like two- and multiple-beam splitters. The ability to transfer condensate population into these kinds of momentum distributions might be useful in designing atom interferometry experiments or for quantum 
(a)
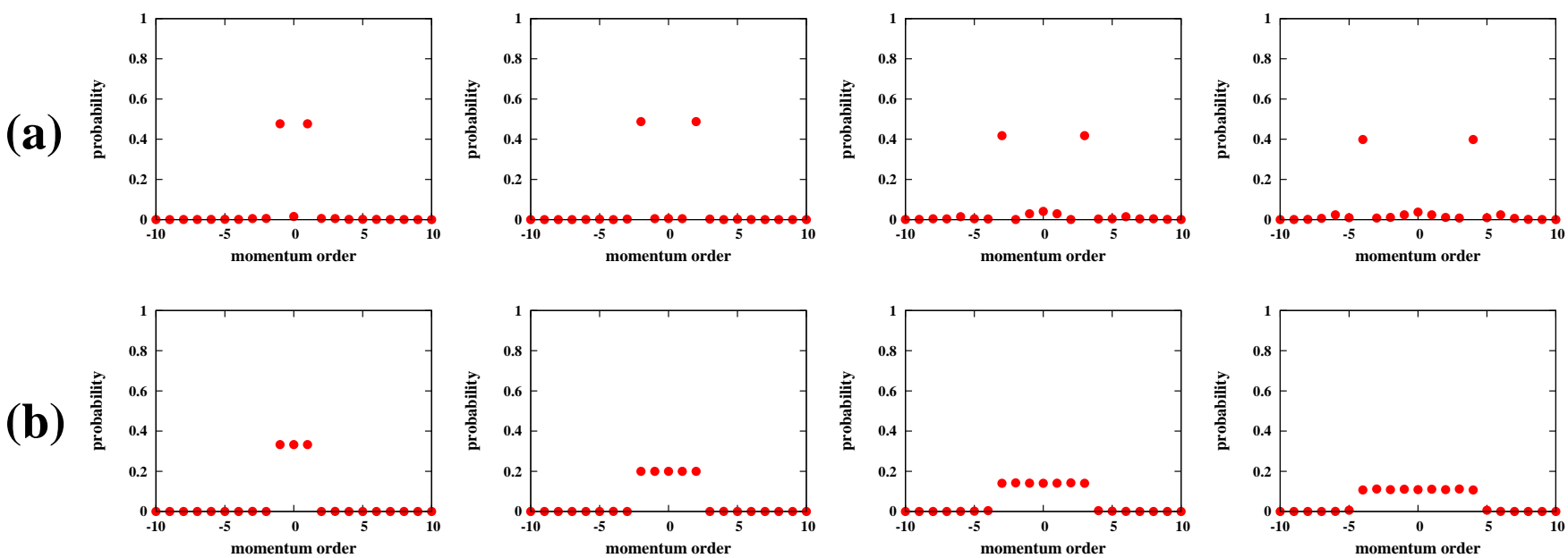

FIG. 4: (color online) These plots show examples of momentum distributions shaped by three pulses. The examples shown are the same as those shown in Fig. 3 for two pulses. The shaping parameters are the areas of the three pulses, $\alpha_{1}, \alpha_{2}$, and $\alpha_{3}$, and the intervals between them, $\beta_{1}$ and $\beta_{2}$. All of the plots above show the probabilities of atoms being in momentum orders $2 m \hbar k$ where $-10 \leq m \leq 10$. (a) The four plots in the top panel exhibit (from left to right) the distributions $D_{m a g}^{(3)}(m)$ where the two momentum states $-2 m \hbar k$ and $+2 m \hbar k$ are equally populated and where $1 \leq m \leq 4$. (b) The four plots in the bottom panel depict (again from left to right) distributions $D_{\text {range }}^{(3)}(m)$ where all of the states in the range between $-2 m \hbar k$ and $+2 m \hbar k$ are equally populated and where $1 \leq m \leq 4$.

information processing. In this regard we are particularly interested in discovering how high a "fidelity" can be achieved with just a few pulses.

Hereafter we will take the measure of the "fidelity," that is, how close the actual momentum probability distribution is to the specified one, to be the minimum value of the least-squares cost function. Thus,

$$
\left(F_{L S}^{(N)}\right)_{\text {min }} \equiv F_{L S}^{(N)}\left(\boldsymbol{\alpha}^{m i n}, \boldsymbol{\beta}^{m i n}\right)
$$

will be used to measure the fidelity.

\section{B. Two-pulse momentum distributions}

For two pulses, the relevant parameters are the dimensionless pulse areas, $\alpha_{1}$ and $\alpha_{2}$, and the interval between the pulses, $\beta_{1}$, measured in units of the Talbot time. We obtained least-squares-designed two-pulse sequences (which we shall refer to as optimal distributions) for eight different specified momentum distributions. These were four momentum magnitude distributions whose specified probabilities are: $D_{\text {mag }}^{(2)}(1): p_{-1}=p_{1}=1 / 2, D_{\text {mag }}^{(2)}(2)$ : $p_{-2}=p_{2}=1 / 2, D_{m a g}^{(2)}(3): p_{-3}=p_{3}=1 / 2$, and $D_{\text {mag }}^{(2)}(4): p_{-4}=p_{4}=1 / 2$. As well as four momentum range distributions: $D_{\text {range }}^{(2)}(1): p_{-1}=p_{0}=p_{1}=1 / 3$, $D_{\text {range }}^{(2)}(2): p_{-2}=\ldots=p_{2}=1 / 5, D_{\text {range }}^{(2)}(1): p_{-3}=$ $p_{0}=p_{3}=1 / 7, D_{\text {range }}^{(2)}(1): p_{-4}=p_{0}=p_{4}=1 / 9$. The results obtained for all of the optimal distributions in both the two-pulse and three-pulse cases are given in Table @ and in Figs. 3 and 4.
Figure 3(a) shows graphs of two-pulse momentum magnitude distributions ordered from left to right along the top row. Each graph exhibits the probability for atoms to be in each momentum order versus of momentum orders $-5 \leq m \leq 5$. The values of the optimal twopulse-sequence parameters and probabilities for each distribution are given in Table [1. As can be seen from the figure, even with two pulses, a remarkably high degree of fidelity with the desired momentum distribution can be obtained with just two pulses. For distribution $D_{\text {mag }}^{(2)}(1), 43.3 \%$ of the population is found in the $m=1$ and $m=-1$ for a total of $86.6 \%$ populating momentum magnitude $2 \hbar k$. For distribution $D_{\text {mag }}^{(2)}(2)$ we find $42.7 \%$ of the population in $m=2$ and $m=-2$ states and $35.7 \%$ in $m= \pm 3$ (distribution $D_{m a g}^{(2)}(3)$ ). The maximum population achievable for two pulses degrades to $28.3 \%$ for $m= \pm 4$. We note that this is far better than is possible for a single pulse where the probability to populate states $\pm m$ is $J_{m}^{2}(\alpha)$. In this case, the maximum values are $33.9 \%(m= \pm 1), 23.7 \%(m= \pm 2), 18.9 \%(m= \pm 3)$, and $16.0 \%(m= \pm 4)$.

Two pulses do remarkably well in producing equal populations in a range of momentum states. Plots of the optimal distributions determined by least-squares method are shown in Fig. 3(b) and column two of Table प gives the values of probabilities achieved for two pulses. One can see that, for distribution $D_{\text {range }}^{(2)}(1)$, the populations in states $m=-1,0,1$ for distribution $D_{\text {range }}^{(2)}(1)$ are the approximately the same, $p_{0}^{(o p t)} \approx p_{1}^{(o p t)}=0.3326$, to four decimal places. This is reasonably close to the specified value of 0.3333 . In distribution $D_{\text {range }}^{(2)}(2)$, the popula- 
TABLE I: The table below presents the optimal values of the parameters and the values of the resulting momentum distribution probabilities for two- and three-pulse sequences for the momentum magnitude and momentum range distributions contained in Figs. 3 and 4 These values were determined by the least-squares procedure discussed in the text. The leftmost column lists the specified momentum distributions in boldface. The next five columns exhibit the parameter values for two-pulse sequences while the final seven columns give the three-pulse-sequence results. The two-pulse parameters are the pulse areas, $\alpha_{1}$ and $\alpha_{2}$, and the interval, $\beta_{1}$, expressed in units of the Talbot time. The three-pulse parameters are the areas of the three pulses, $\alpha_{1}$,

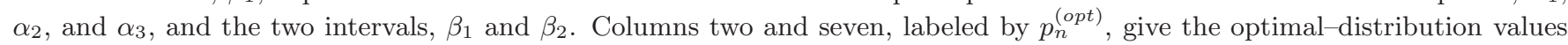
of the nonzero specified probabilities. The probability given is listed in the first column in lightface type. Columns six and thirteen list the fidelity of the least-squares momentum distributions to the specified distribution by giving the value of the least-squares functions $F_{L S}^{(2)}$ and $F_{L S}^{(3)}$ evaluated at the values of the parameters listed in the table.

\begin{tabular}{|c|c|c|c|c|c|c|c|c|c|c|c|c|}
\hline \multirow{2}{*}{$\begin{array}{l}\text { Momentum } \\
\text { Distribution }\end{array}$} & \multicolumn{5}{|c|}{ Two-pulse results } & \multicolumn{7}{|c|}{ Three-pulse results } \\
\hline & $p_{n}^{(o p t)}$ & $\alpha_{1}^{\min }$ & $\beta_{1}^{\min }$ & $\alpha_{2}^{\min }$ & $\left(F_{L S}^{(2)}\right)_{\min }$ & $p_{n}^{(o p t)}$ & $\alpha_{1}^{\min }$ & $\beta_{1}^{\text {min }}$ & $\alpha_{2}^{\min }$ & $\beta_{2}^{\min }$ & $\alpha_{3}^{\min }$ & $\left(F_{L S}^{(3)}\right)_{\min }$ \\
\hline $\boldsymbol{p}_{-1}=\boldsymbol{p}_{1}=\frac{1}{2}$ & & 1.715 & 0.130 & 0.594 & $1.5 \times 10^{-2}$ & & 3.737 & 0.481 & 3.402 & 0.453 & 1.782 & $1.9 \times 10^{-3}$ \\
\hline$p_{-1}^{(o p t)}=p_{1}^{(o p t)}$ & 0.4327 & & & & & 0.4766 & & & & & & \\
\hline $\boldsymbol{p}_{-2}=\boldsymbol{p}_{2}=\frac{1}{2}$ & & 2.857 & 0.320 & 1.04 & $1.4 \times 10^{-2}$ & & 2.733 & 0.342 & 0.585 & 0.337 & 0.882 & $3.9 \times 10^{-4}$ \\
\hline$p_{-2}^{(o p t)}=p_{2}^{(o p t)}$ & 0.4271 & & & & & 0.4877 & & & & & & \\
\hline $\boldsymbol{p}_{-3}=\boldsymbol{p}_{3}=\frac{1}{2}$ & & 3.560 & 0.378 & 1.429 & $5.6 \times 10^{-2}$ & & 1.297 & 0.586 & 7.347 & 0.404 & 2.310 & $1.7 \times 10^{-2}$ \\
\hline$p_{-3}^{(o p t)}=p_{3}^{(o p t)}$ & 0.3572 & & & & & 0.4179 & & & & & & \\
\hline $\boldsymbol{p}_{-4}=\boldsymbol{p}_{4}=\frac{1}{2}$ & & 4.230 & 0.408 & 1.790 & $1.2 \times 10^{-1}$ & & 3.158 & 0.376 & 2.936 & 0.287 & 1.047 & $2.5 \times 10^{-2}$ \\
\hline$p_{-4}^{(o p t)}=p_{4}^{(o p t \bar{t}}$ & 0.2833 & & & & & 0.3984 & & & & & & \\
\hline $\boldsymbol{p}_{-1}=\boldsymbol{p}_{0}=\boldsymbol{p}_{1}=\frac{1}{3}$ & & 1.075 & 0.153 & 0.524 & $3.6 \times 10^{-6}$ & & 0.551 & 0.565 & 1.676 & 0.856 & 0.547 & $1.4 \times 10^{-6}$ \\
\hline$p_{0}^{(o p t)}$ & 0.3326 & & & & & 0.3328 & & & & & & \\
\hline$p_{-1}^{(o p t)}=p_{1}^{(o p t)}$ & 0.3326 & & & & & 0.3328 & & & & & & \\
\hline $\boldsymbol{p}_{-2}=\ldots=\boldsymbol{p}_{2}=\frac{1}{5}$ & & 1.861 & 0.306 & 0.468 & $5.6 \times 10^{-6}$ & & 1.730 & 0.058 & 0.206 & 0.252 & 0.491 & $5.0 \times 10^{-7}$ \\
\hline$p_{0}^{(o p t)}$ & 0.1994 & & & & & 0.1998 & & & & & & \\
\hline$p_{-1}^{(o p t)}=p_{1}^{(o p t)}$ & 0.1994 & & & & & 0.1998 & & & & & & \\
\hline$p_{-2}^{(o p t)}=p_{2}^{(o p t)}$ & 0.1993 & & & & & 0.1998 & & & & & & \\
\hline $\boldsymbol{p}_{-3}=\ldots=\boldsymbol{p}_{3}=\frac{1}{7}$ & & 2.416 & 0.394 & 0.836 & $2.4 \times 10^{-3}$ & & 2.205 & 0.451 & 0.739 & 0.260 & 2.112 & $6.7 \times 10^{-5}$ \\
\hline$p_{0}^{(o p t)}$ & 0.1370 & & & & & 0.1402 & & & & & & \\
\hline$p_{-1}^{(o p t)}=p_{1}^{(o p t)}$ & 0.1276 & & & & & 0.1410 & & & & & & \\
\hline$p_{-2}^{(o p t)}=p_{2}^{(o p t)}$ & 0.1605 & & & & & 0.1423 & & & & & & \\
\hline$p_{-3}^{(o p t)}=p_{3}^{(o p t)}$ & 0.1248 & & & & & 0.1405 & & & & & & \\
\hline $\boldsymbol{p}_{-4}=\ldots=\boldsymbol{p}_{4}=\frac{1}{9}$ & & 0.849 & 0.218 & 3.895 & $4.8 \times 10^{-3}$ & & 2.436 & 0.267 & 1.627 & 0.402 & 0.949 & $1.3 \times 10^{-4}$ \\
\hline$p_{0}^{(o p t)}$ & 0.0869 & & & & & 0.1087 & & & & & & \\
\hline$p_{-1}^{(o p t)}=p_{1}^{(o p t)}$ & 0.1105 & & & & & 0.1108 & & & & & & \\
\hline$p_{-2}^{(o p t)}=p_{2}^{(o p t)}$ & 0.1034 & & & & & 0.1087 & & & & & & \\
\hline$p_{-3}^{(o p t)}=p_{3}^{(o p t)}$ & 0.1323 & & & & & 0.1120 & & & & & & \\
\hline$p_{-4}^{(o p t)}=p_{4}^{(o p t)}$ & 0.0802 & & & & & 0.1073 & & & & & & \\
\hline
\end{tabular}

tions in states $m=-2,-1,0,1,2$ the distribution across these states vary between 0.1993 and 0.1994 which compares well with the specified value of 0.2 . For distribution, $D_{\text {range }}^{(2)}(3)$, while the states $m=-3,-2,-1,0,1,2,3$ contain more than $96 \%$ of the total population, the probabilities vary between 0.1248 and 0.1605 producing a relatively large variance around the specified value of $1 / 7=0.1429$. The degradation of the fidelity can especially be seen in distribution $D_{\text {range }}^{(2)}(4)$ both in the value of $\left(F_{L S}^{(2)}\right)_{\text {min }}$ and in the deviation of the achievable probabilities from the specified probability as shown in Table [1. For this distribution, the probabilites vary between 0.0802 and 0.1323 and deviate significantly from the specified value of $1 / 9=0.1111$.
Upon closer inspection, we found that the optimal twopulse sequences had the common characteristic that there was a single dominant pathway to each of the prescribed final momentum states. Thus, for a fixed final momentum state, this dominant pathway consisted of a direct jump from the zero-momentum original condensate to the final momentum at the first pulse followed by evolution between the pulses and no jump in momentum at the second pulse. In this single-dominant-pathway picture, the total amplitude is somewhat insensitive to the time between pulses since the coherent sum can be (roughly) approximated with a single term. 


\section{Three-pulse momentum distributions}

For three pulses, the relevant parameters are the pulse areas, $\alpha_{1}, \alpha_{2}$, and $\alpha_{3}$, and the intervals between the pulses, $\beta_{1}$ and $\beta_{2}$. We obtained optimal three-pulse sequences for the same set of eight specified momentum distributions as for two pulses. As can be seen in Fig. 4. the fidelities achievable with three pulses is better than with two pulses and is far superior to the single pulse case.

Figure 4(a) (top row) shows three-pulse momentum magnitude distributions $D_{\text {mag }}^{(3)}(1), D_{\text {mag }}^{(3)}(2), D_{\text {mag }}^{(3)}(3)$, and $D_{\text {mag }}^{(3)}(4)$ respectively from left to right. While the bottom row shows three-pulse momentum range distributions, $D_{\text {range }}^{(3)}(1), D_{\text {range }}^{(3)}(2), D_{\text {range }}^{(3)}(3)$, and $D_{\text {range }}^{(3)}(4)$ again from left to right. These plots show that the threepulse optimal distributions faithfully reproduce the specified distributions better than the two-pulse versions. Comparisons of the fidelities for the two-pulse case versus the three-pulse given in Table \ shows that three pulse does a better job at reproducing the specified distribution in every case.

For some of the distributions there is little difference between two pulses and three pulses because of the high fidelity of the two-pulse case. One example is $D_{\text {range }}^{(2)}(1)$ versus $D_{\text {range }}^{(3)}(1)$. However, three pulses is clearly better for the $D_{\text {range }}^{(3)}(3)$ and $D_{\text {range }}^{(3)}(4)$ distributions.

\section{CONCLUSION}

In this paper we have shown that it is possible to create clouds of coherent atoms with momentumspace distributions important for applications in atominterferometry with a sequence of only two or three standing-wave laser pulses applied to a BEC. We derived the momentum distribution for atoms in a BEC after $N$ short-time, standing-wave laser pulses were applied where the pulse areas and time intervals between the pulses were variable. This distribution was a generalization of the expression for two pulses whose validity has been verified experimentally [1]. We further described a method for designing pulse sequences that produce a specified momentum distribution of the condensate atoms. We found that two kinds of distributions that have important applications as beam splitters can be produced with high fidelity with two or three pulses. We also found that the optimal two-pulse sequences obtained could be understood in terms of a single-dominant-pathway picture.

The ability to produce coherent atom with engineered momentum-space distributions can now become a new tool for the design of new atom interferometer schemes. Methods for initial momentum-state selection or for producing multiple-beam splitters can now be designed. For example, one could imagine a Bose-Einstein condensate created and confined on an atom chip to which could be applied a sequence of pulses such as $D_{\text {range }}^{(3)}(2)$ so that the cloud is split into five equal parts. If such a condensate were confined by a harmonic trap potential, these parts would eventually all come back together at once where they could be split again. This would produce multiple interference patterns that reflect the different phase evolutions along the different pathways. Such multi-particle interferometers could, in principle, implement quantum computations or be used for precision navigation applications, gradiometry, or fundamental studies.

\section{Appendix A}

This appendix presents the derivation of Eq. (9) which expresses the condensate wave function just after the application of a short-time, standing-wave laser pulse in terms of the wave function just before the pulse.

We begin by transforming away the internal energies of the atom:

$$
\psi_{k}\left(\mathbf{r}_{a}, t\right) \equiv e^{-i E_{k} t / \hbar} \phi_{k}\left(\mathbf{r}_{a}, t\right) . \quad k=g, e
$$

Under this transformation, Eqs. (7) and (8) become

$$
\begin{aligned}
i \hbar \frac{\partial \phi_{g}}{\partial t} & =H_{0}^{(g)} \phi_{g}\left(\mathbf{r}_{a}, t\right) \\
& +\hbar \Omega_{0} \cos \left(\mathbf{k}_{L} \cdot \mathbf{r}_{a}\right) f(t) e^{-i \Delta t} \phi_{e}\left(\mathbf{r}_{a}, t\right) \\
& +g N\left(\left|\phi_{g}\left(\mathbf{r}_{a}, t\right)\right|^{2}+\left|\phi_{e}\left(\mathbf{r}_{a}, t\right)\right|^{2}\right) \phi_{g}\left(\mathbf{r}_{a}, t\right)
\end{aligned}
$$

and

$$
\begin{aligned}
i \hbar \frac{\partial \phi_{e}}{\partial t} & =H_{0}^{(e)} \phi_{e}\left(\mathbf{r}_{a}, t\right) \\
& +\hbar \Omega_{0} \cos \left(\mathbf{k}_{L} \cdot \mathbf{r}_{a}\right) f(t) e^{i \Delta t} \phi_{g}\left(\mathbf{r}_{a}, t\right) \\
& +g N\left(\left|\phi_{g}\left(\mathbf{r}_{a}, t\right)\right|^{2}+\left|\phi_{e}\left(\mathbf{r}_{a}, t\right)\right|^{2}\right) \phi_{e}\left(\mathbf{r}_{a}, t\right)
\end{aligned}
$$

where we have made the rotating-wave approximation (RWA). The RWA consists of neglecting the exponentials that oscillate as $\exp \left[ \pm i\left(\omega_{0}+\omega_{L}\right) t\right]$ relative to $\exp ( \pm i \Delta t)$ where $\hbar \omega_{0}=E_{e}-E_{g}$. This approximation holds because $\omega_{L}$ is typically six orders of magnitude larger than $\Delta$ for optical frequencies when the light is close to resonance.

Since we assume $\Omega_{0} \ll \Delta$, there is never very much population in the upper state. Thus we can neglect the nonlinear term and kinetic plus trap potential energy terms in Eq. A3 and can neglect $\phi_{e}$ in the nonlinear term of Eq. A2 . This gives

$$
\begin{aligned}
i \hbar \frac{\partial \phi_{g}}{\partial t} & \approx H_{0}^{(g)} \phi_{g}\left(\mathbf{r}_{a}, t\right)+g N\left|\phi_{g}\left(\mathbf{r}_{a}, t\right)\right|^{2} \phi_{g}\left(\mathbf{r}_{a}, t\right) \\
& +\hbar \Omega_{0} \cos \left(\mathbf{k}_{L} \cdot \mathbf{r}_{a}\right) f(t) e^{-i \Delta t} \phi_{e}\left(\mathbf{r}_{a}, t\right)
\end{aligned}
$$




$$
i \hbar \frac{\partial \phi_{e}}{\partial t} \approx \hbar \Omega_{0} \cos \left(\mathbf{k}_{L} \cdot \mathbf{r}_{a}\right) f(t) e^{i \Delta t} \phi_{g}\left(\mathbf{r}_{a}, t\right)
$$

Finally, note that the first two terms on the right-handside of Eq. (A4) approximately satisfy the static GP equation and thus together equal $\mu \phi_{g}$. Replacing those two terms gives:

$$
\begin{aligned}
i \hbar \frac{\partial \phi_{g}}{\partial t} & \approx \mu \phi_{g}\left(\mathbf{r}_{a}, t\right) \\
& +\hbar \Omega_{0} \cos \left(\mathbf{k}_{L} \cdot \mathbf{r}_{a}\right) f(t) e^{-i \Delta t} \phi_{e}\left(\mathbf{r}_{a}, t\right)
\end{aligned}
$$

This term can be transformed away but it is unecessary since we have assumed that $\mu \delta t / \hbar \ll 1$. Neglecting this term finally gives

$$
\begin{aligned}
i \hbar \frac{\partial \phi_{g}}{\partial t} & \approx \hbar \Omega_{0} \cos \left(\mathbf{k}_{L} \cdot \mathbf{r}_{a}\right) f(t) e^{-i \Delta t} \phi_{e}\left(\mathbf{r}_{a}, t\right)( \\
i \hbar \frac{\partial \phi_{e}}{\partial t} & \approx \hbar \Omega_{0} \cos \left(\mathbf{k}_{L} \cdot \mathbf{r}_{a}\right) f(t) e^{i \Delta t} \phi_{g}\left(\mathbf{r}_{a}, t\right)
\end{aligned}
$$

Under the "sudden approximation," these equations can be easily solved. That is, we assume that the turn-on of the pulse is fast enough that the atom remains in its initial state until the pulse is fully on.

In order to express the solution in terms of the area of a single pulse, we transform the solutions as

$$
\left(\begin{array}{c}
\phi_{g}\left(\mathbf{r}_{a}, t\right) \\
\phi_{e}\left(\mathbf{r}_{a}, t\right)
\end{array}\right)=\left(\begin{array}{cc}
e^{-i \Delta t / 2} & 0 \\
0 & e^{i \Delta t / 2}
\end{array}\right)\left(\begin{array}{c}
\bar{\phi}_{g}\left(\mathbf{r}_{a}, t\right) \\
\bar{\phi}_{e}\left(\mathbf{r}_{a}, t\right)
\end{array}\right)
$$

This yields the following equations for $\bar{\phi}_{g, e}$ :

$$
\begin{aligned}
i \hbar \frac{\partial \bar{\phi}_{g}}{\partial t} & =-\frac{1}{2} \hbar \Delta \bar{\phi}_{g}\left(\mathbf{r}_{a}, t\right)+V\left(\mathbf{r}_{a}, t\right) \bar{\phi}_{e}\left(\mathbf{r}_{a}, t\right) \\
i \hbar \frac{\partial \bar{\phi}_{e}}{\partial t} & =\frac{1}{2} \hbar \Delta \bar{\phi}_{e}\left(\mathbf{r}_{a}, t\right)+V\left(\mathbf{r}_{a}, t\right) \bar{\phi}_{g}\left(\mathbf{r}_{a}, t\right)(\mathrm{A} 10)
\end{aligned}
$$

where

$$
V\left(\mathbf{r}_{a}, t\right)=\hbar \Omega_{0} f(t) \cos \left(\mathbf{k}_{L} \cdot \mathbf{r}_{a}\right) .
$$

Defining

$$
\bar{\Phi}\left(\mathbf{r}_{a}, t\right) \equiv\left(\begin{array}{c}
\bar{\phi}_{g}\left(\mathbf{r}_{a}, t\right) \\
\bar{\phi}_{e}\left(\mathbf{r}_{a}, t\right)
\end{array}\right)
$$

and

$$
\mathcal{H}\left(\mathbf{r}_{a}, t\right) \equiv\left(\begin{array}{cc}
-\frac{1}{2} \hbar \Delta & V\left(\mathbf{r}_{a}, t\right) \\
V\left(\mathbf{r}_{a}, t\right) & \frac{1}{2} \hbar \Delta
\end{array}\right)
$$

we may formally express Eqs. A10 as

$$
i \hbar \frac{\partial \bar{\Phi}}{\partial t}=\mathcal{H}\left(\mathbf{r}_{a}, t\right) \bar{\Phi}\left(\mathbf{r}_{a}, t\right) .
$$

Since $\mathcal{H}\left(\mathbf{r}_{a}, t\right)$ is constant during the pulse we obtain a relationship between $\bar{\Phi}$ before and after the pulse

$$
\bar{\Phi}\left(\mathbf{r}_{a}, t_{0}+\delta t / 2\right)=e^{-i M \delta t} \bar{\Phi}\left(\mathbf{r}_{a}, t_{0}-\delta t / 2\right),
$$

where

$$
M=\left(\begin{array}{cc}
-\frac{1}{2} \Delta & \Omega_{0} \cos \left(\mathbf{k}_{L} \cdot \mathbf{r}_{a}\right) \\
\Omega_{0} \cos \left(\mathbf{k}_{L} \cdot \mathbf{r}_{a}\right) & \frac{1}{2} \Delta
\end{array}\right) .
$$

This matrix can be easily exponentiated using its eigenvalues,

$$
\lambda_{ \pm}= \pm\left[\left(\frac{1}{2} \Delta\right)^{2}+\Omega_{0}^{2} \cos ^{2}\left(\mathbf{k}_{L} \cdot \mathbf{r}_{a}\right)\right]^{1 / 2} \equiv \pm \lambda,
$$

and eigenvectors

$$
|+\lambda\rangle=\left(\begin{array}{c}
\sin (\theta / 2) \\
\cos (\theta / 2)
\end{array}\right) \quad|-\lambda\rangle=\left(\begin{array}{c}
\cos (\theta / 2) \\
-\sin (\theta / 2)
\end{array}\right),
$$

where $\theta$ is defined by

$$
\sin (\theta)=\frac{\Omega_{0} \cos \left(\mathbf{k}_{L} \cdot \mathbf{r}_{a}\right)}{\lambda}, \quad \cos (\theta)=\frac{\frac{1}{2} \Delta}{\lambda} .
$$

The diagonalization matrix, $U^{\dagger}$, where

$$
M_{D}=U M U^{\dagger}=\left(\begin{array}{cc}
\lambda & 0 \\
0 & -\lambda
\end{array}\right) \text {, }
$$

is given by

$$
U^{\dagger}=\left(\begin{array}{cc}
\sin (\theta / 2) & \cos (\theta / 2) \\
\cos (\theta / 2) & -\sin (\theta / 2)
\end{array}\right)
$$

The exponentiated matrix is, therefore

$$
e^{-i M \delta t}=\left(\begin{array}{cc}
s^{2} e^{-i \lambda \delta t}+c^{2} e^{i \lambda \delta t} & -s c\left(e^{i \lambda \delta t}-e^{-i \lambda \delta t}\right) \\
-s c\left(e^{i \lambda \delta t}-e^{-i \lambda \delta t}\right) & c^{2} e^{-i \lambda \delta t}+s^{2} e^{i \lambda \delta t}
\end{array}\right)
$$

where $s \equiv \sin (\theta / 2)$ and $c \equiv \cos (\theta / 2)$.

Thus we can write a relationship between $\bar{\phi}_{g}$ before and after the pulse. From Eqs. A15 and (A22) we have

$$
\begin{aligned}
\bar{\phi}_{g}\left(\mathbf{r}_{a}, t_{+}\right)= & \left(s^{2} e^{-i \lambda \delta t}+c^{2} e^{i \lambda \delta t}\right) \bar{\phi}_{g}\left(\mathbf{r}_{a}, t_{-}\right) \\
& -s c\left(e^{i \lambda \delta t}-e^{-i \lambda \delta t}\right) \bar{\phi}_{e}\left(\mathbf{r}_{a}, t_{-}\right)
\end{aligned}
$$

where $t_{ \pm} \equiv t_{0} \pm \delta t / 2$.

We can simplify the above expression by invoking the approximation that the detuning from resonance is much larger that the single-photon Rabi frequency, $\Omega_{0} \ll \Delta$. In this case, we have

$$
\begin{aligned}
\lambda & \approx \frac{1}{2} \Delta+\left(\frac{\Omega_{0}^{2}}{\Delta}\right) \cos ^{2}\left(\mathbf{k}_{L} \cdot \mathbf{r}_{a}\right) \\
& =\frac{1}{2} \Delta+\frac{1}{2}\left(\frac{\Omega_{0}^{2}}{\Delta}\right)\left(1+\cos \left(2 \mathbf{k}_{L} \cdot \mathbf{r}_{a}\right)\right)
\end{aligned}
$$


Furthermore,

$$
\begin{aligned}
\cos (\theta) & =\frac{\frac{1}{2} \Delta}{\lambda} \\
& =\frac{\frac{1}{2} \Delta}{\left(\left(\frac{1}{2} \Delta\right)^{2}+\Omega_{0}^{2} \cos ^{2}\left(\mathbf{k}_{L} \cdot \mathbf{r}_{a}\right)\right)^{1 / 2}} \\
& \approx 1-\frac{1}{2}\left(\frac{\Omega_{0}}{\frac{1}{2} \Delta}\right)^{2} \cos ^{2}\left(\mathbf{k}_{L} \cdot \mathbf{r}_{a}\right)
\end{aligned}
$$

The factors $s^{2}$ and $c^{2}$ are, to second order in $\Omega_{0} / \Delta$, approximately

$$
\begin{aligned}
s^{2} & \equiv \sin ^{2}(\theta / 2) \\
& =\frac{1}{2}(1-\cos (\theta))=\frac{1}{2}\left(1-\frac{\frac{1}{2} \Delta}{\lambda}\right) \\
s^{2} & \approx\left(\frac{\Omega_{0}}{\Delta}\right)^{2} \cos ^{2}\left(\mathbf{k}_{L} \cdot \mathbf{r}_{a}\right) \\
c^{2} & \equiv \cos ^{2}(\theta / 2) \\
& =\frac{1}{2}(1+\cos (\theta))=\frac{1}{2}\left(1+\frac{\frac{1}{2} \Delta}{\lambda}\right) \\
c^{2} & \approx 1-\left(\frac{\Omega_{0}}{\Delta}\right)^{2} \cos ^{2}\left(\mathbf{k}_{L} \cdot \mathbf{r}_{a}\right) .
\end{aligned}
$$

Given the above it should be an excellent approximation to set $s \approx 0$ and $c \approx 1$. Hence

$$
\bar{\phi}_{g}\left(\mathbf{r}_{a}, t_{+}\right) \approx e^{i\left(\frac{1}{2} \Delta+\Omega_{2}\right) \delta t+i \Omega_{2} \delta t \cos \left(2 \mathbf{k}_{L} \cdot \mathbf{r}_{a}\right)} \bar{\phi}_{g}\left(\mathbf{r}_{a}, t_{-}\right),
$$

where

$$
\Omega_{2} \equiv \frac{\Omega_{0}^{2}}{2 \Delta}
$$

is the two-photon Rabi frequency. We can express the above relationship in terms of the original condensate wave function as

$$
\begin{aligned}
\phi_{g}\left(\mathbf{r}_{a}, t_{+}\right) \approx & e^{i \Omega_{2} \delta t} \\
& \times e^{i \Omega_{2} \delta t \cos \left(2 \mathbf{k}_{L} \cdot \mathbf{r}_{a}\right)} \phi_{g}\left(\mathbf{r}_{a}, t_{-}\right) .
\end{aligned}
$$

This is the effect of a single standing-wave pulse on the condensate wave function.

\section{Acknowledgments}

We acknowledge helpful discussions with L. Deng, E.W. Hagley, I. Spielman, A. Cassidy, G. Campbell, and W.D. Phillips. This work was supported in part by the U.S. National Science Foundation grant PHY-0758111, the Physics Frontiers Center grant PHY-0822671 and by the National Institute of Standards and Technology.
[1] M. Anderson, J. Ensher, M. Matthews, C. Wieman, and E. Cornell, Science 269, 198 (1995).

[2] C. C. Bradley, C. A. Sackett, J. J. Tollett, and R. G. Hulet, Phys. Rev. Lett. 75, 1687 (1995).

[3] K. B. Davis, M. O. Mewes, M. R. Andrews, N. J. van Druten, D. S. Durfee, D. M. Kurn, and W. Ketterle, Phys. Rev. Lett. 75, 3969 (1995).

[4] C. Pethick and H. Smith, Bose-Einstein Condensation in Dilute Gases (Cambridge University Press, 2008), 2nd ed.

[5] L. Pitaevskii and S. Stringari, Bose-Einstein Condensation (Oxford University Press, 2003).

[6] M. S. Chapman, C. R. Ekstrom, T. D. Hammond, R. A. Rubenstein, J. Schmiedmayer, S. Wehinger, and D. E. Pritchard, Phys. Rev. Lett. 74, 4783 (1995).

[7] L. Hackermüller, S. Uttenthaler, K. Hornberger, E. Reiger, B. Brezger, A. Zeilinger, and M. Arndt, Phys. Rev. Lett. 91, 090408 (2003).

[8] S. Gerlich, M. Gring, H. Ulbricht, K. Hornberger, J. Tüxen, M. Mayor, and M. Arndt, Angew. Chem. Int. Ed. 47, 6195 (2008).

[9] M.-O. Mewes, M. R. Andrews, D. M. Kurn, D. S. Durfee, C. G. Townsend, and W. Ketterle, Phys. Rev. Lett. 78, 582 (1997).

[10] I. Bloch, T. W. Hänsch, and T. Esslinger, Phys. Rev. Lett. 82, 3008 (1999).

[11] L. Deng, E. W. Hagley, J. Denschlag, J. E. Simsarian, M. Edwards, C. W. Clark, K. Helmerson, S. L. Rolston, and W. D. Phillips, Phys. Rev. Lett. 83, 5407 (1999).
[12] S. M. Tan and D. F. Walls, Phys. Rev. A 47, 4663 (1993).

[13] A. Peters, K. Chung, and S. Chu, Metrologia 38, 25 (2001).

[14] J. M. McGuirk, G. T. Foster, J. B. Fixler, M. J. Snadden, and M. A. Kasevich, Phys. Rev. A 65, 033608 (2002).

[15] T. Gustavson, P. Bouyer, and M. Kasevich, Classical Quantum Gravity 17, 2385 (2000).

[16] A. Miffre, M. Jacquey, M. Buchner, G. Trenec, and J. Vigué, European Journal of Physics D 38, 353 (2006).

[17] A. D. Cronin, J. Schmiedmayer, and D. E. Pritchard, Rev. Mod. Phys. 81, 1051 (2009).

[18] Y.-J. Wang, D. Z. Anderson, V. M. Bright, E. A. Cornell, Q. Diot, T. Kishimoto, M. Prentiss, R. A. Saravanan, S. R. Segal, and S. Wu, Phys. Rev. Lett. 94, 090405 (2005).

[19] S. Wu, Y.-J. Wang, Q. Diot, and M. Prentiss, Phys. Rev. A 71, 043602 (2005).

[20] P. R. Berman, B. Dubetsky, and J. L. Cohen, Phys. Rev. A 58, 4801 (1998).

[21] P. R. Berman and B. Bian, Phys. Rev. A 55, 4382 (1997).

[22] V. S. Malinovsky and P. R. Berman, Phys. Rev. A 68, 023610 (2003).

[23] F. W. J. Olver, D. W. Lozier, R. F. Boisvert, and C. W. Clark, eds., NIST Handbook of Mathematical Functions (Cambridge University Press, 2010).

[24] W. Petrich, M. H. Anderson, J. R. Ensher, and E. A. Cornell, Phys. Rev. Lett. 74, 3352 (1995). 